\title{
Follicular dendritic cell dysfunction contributes to impaired antigen-specific humoral responses in sepsis-surviving mice
}

\author{
Minakshi Rana, ${ }^{1}$ Andrea La Bella, ${ }^{1}$ Rivka Lederman, ${ }^{1}$ Bruce T. Volpe, ${ }^{2}$ Barbara Sherry, ${ }^{3}$ and Betty Diamond ${ }^{1}$ \\ 'Center for Autoimmune, Musculoskeletal and Hematopoietic Diseases, ${ }^{2}$ Center for Biomedical Science, and ${ }^{3}$ Center for Immunology and Inflammation, Feinstein Institutes for Medical Research, \\ New York, New York, USA.
}

\begin{abstract}
Sepsis survivors exhibit impaired responsiveness to antigen $(\mathrm{Ag})$ challenge associated with increased mortality from infection. The contribution of follicular dendritic cells (FDCs) in the impaired humoral response in sepsis-surviving mice is investigated in this study. We demonstrated that mice subjected to sepsis from cecal ligation and puncture (CLP mice) have reduced NP-specific high-affinity class-switched Ig antibodies (Abs) compared with sham-operated control mice following immunization with the T cell-dependent Ag, NP-CGC. NP-specific germinal center (CC) B cells in CLP mice exhibited reduced TNF- $\alpha$ and AID mRNA expression compared with sham-operated mice. CLP mice showed a reduction in FDC clusters, a reduced binding of immune complexes on FDCs, and reduced mRNA expression of CR2, ICAM-1, VCAM-1, Fc $\gamma R$ IIB, TNFR1, IKK2, and LT $\beta R$ compared with sham-operated mice. Adoptive transfer studies showed that there was no B cell-intrinsic defect. In summary, our data suggest that the reduced $\mathrm{Ag}$-specific $\mathrm{Ab}$ response in CLP mice is secondary to a disruption in FDC and CC B cell function.
\end{abstract}

\section{Introduction}

Sepsis is defined as life-threatening organ dysfunction resulting from a dysregulated host response to infection. It is a major cause of death in the United States, accounting for billions of dollars in hospital costs $(1,2)$. Despite advancements in critical care medicine, most sepsis-surviving patients die within 5 years of their septic episode because of persistent immune suppression (3-6). Sepsis-induced immune alterations affect both innate and adaptive immune responses (7).

Natural infection and vaccinations elicit antibody $(\mathrm{Ab})$ production by $\mathrm{B}$ cells, providing a critical line of defense against invading microbes (8). Ab responses can be categorized as $\mathrm{T}$ celldependent (TD) or T cell-independent (TI) based on the need for CD4 T cell help (9). B cells in TD responses can adopt one of two fates: (a) extrafollicular B cell activation generating short-lived plasma cells (PCs) or (b) a germinal center (GC) response generating B cell memory and long-lived PCs (LLPCs; refs. 10, 11). In the classical GC reaction, antigen-activated (Ag-activated) B cells in the dark zone undergo rapid rounds of proliferation and somatic hypermutation (SHM) of their Ig-variable region genes and migrate to the light zone, where selection for B cells with increased affinity for Ag occurs (12-14). Follicular dendritic cells (FDCs) are radioresistant nonhematopoietic stromal cells that form the nidus for the GC response (15). The selection process in the light zone involves competition among GC B cells for capture of native Ag in the form of immune complexes (ICs) displayed on FDCs $(12,13$,

Conflict of interest: The authors have declared that no conflict of interest exists. Copyright: (5) 2021, American Society for Clinical Investigation.

Submitted: December 9, 2020; Accepted: April 29, 2021; Published: June 15, 2021.

Reference information: J Clin Invest. 2021;131(12):e146776.

https://doi.org/10.1172/JCl146776.
16). FDCs express complement receptors (CRs) and Fc receptors, which allow them to trap and retain ICs $(17,18)$. High-affinity B cells receive survival and proliferation signals from FDCs, whereas low-affinity $\mathrm{B}$ cells fail to receive these signals and undergo apoptosis (19). High-affinity GC B cells internalize Ag and process and display antigenic peptides to $\mathrm{T}$ follicular helper (Tfh) cells in peptide-MHCII complexes, thereby receiving additional Tfhderived survival signals (15).

It is well-documented that sepsis leads to impaired adaptive immune responses, including reduced Ag-specific humoral responses (20-22). B and $\mathrm{T}$ cell defects have been demonstrated in sepsis mice (21-23), but there are some less well-examined cell types, including FDCs, the dysfunction of which may also contribute to the overall defect. Some reports show that FDCs undergo apoptosis at the onset of sepsis through a caspase-3-mediated mechanism $(24,25)$. However, the contribution of FDC dysfunction to the long-term impairment in $\mathrm{Ab}$ responses has not been explored.

The current study focused on the role of FDCs in mediating impaired Ag-specific humoral responses in mice surviving sepsis induced by cecal ligation and puncture (CLP mice). We demonstrate that in the CLP model of polymicrobial sepsis of gastrointestinal origin, Ag-specific GC responses are impaired. CLP mice showed compromised generation of NP-specific high-affinity class-switched Abs, due to defective $\mathrm{B}$ cell differentiation, and reduced PC and memory B cell formation. Adoptive transfer studies showed that these impairments were not B cell intrinsic.

CLP mice exhibited a reduced number of FDCs clusters, reduced retention of ICs on the FDCs, and altered FDC gene expression, suggesting that FDCs may be functionally compromised in CLP mice. Our results suggest that FDC dysfunction may be an appropriate therapeutic target to restore humoral immune responses in sepsis survivors. 


\section{Results}

$T D A b$ responses are impaired in CLP survivors. Polymicrobial sepsis was induced by using a CLP model. The perforation of cecum releases fecal matter into the peritoneal cavity to generate polymicrobial infection. Approximately $30 \%$ of clinical sepsis is of gastrointestinal origin (26). Sepsis-surviving mice showed a progressive clearance of bacteremia, such that 21 days after CLP no bacteria were detectable in blood in all mice examined $(n=7)$. Bacteria were still detected in the peritoneal cavity at day 22, but by day 28 after CLP when immunizations were performed, no bacteria were detectable in 3 of 4 CLP mice examined. To confirm previous studies $(20,21)$ demonstrating a blunted Ag-specific humoral response in sepsis-surviving mice, we immunized sham-operated (sham) and CLP mice with the TD Ag, (4-hydroxy-3-nitrophenyl) acetyl (NP) chicken $\gamma$-globulin (CGG) (NP-CGG), in alum 4 weeks after surgery. The experimental approach is shown in Figure 1A. CLP mice exhibited minimal induction of both low-affinity $\left(\mathrm{NP}_{23}\right.$; Figure 1, B-D) and high-affinity ( $\mathrm{NP}_{2}$; Figure 1, E-G) anti-NP-IgG, -IgG1, and -IgG2c Abs compared with sham mice at day 7 after primary immunization (Figure $1, A^{-} G$ ). This was not merely a delayed response, as titers failed to rise on days 14, 21, and 28. Our results suggest that B cells in CLP mice fail to mount a GC response.

We also tested the TI response to NP by immunizing mice with NP-ficoll. NP-ficoll-immunized CLP mice produced enhanced serum anti-NP-IgM and -IgG Abs in response to TI immunization, compared with sham mice (Figure 1, H and I), demonstrating that the defect in humoral responses in CLP mice is limited to the response to TD. Of note, CLP mice exhibited an increase in total serum IgG compared with sham mice (Supplemental Figure 1 ; supplemental material available online with this article; https:// doi.org/10.1172/JCI146776DS1), suggesting that the Ag-specific response is impaired after sepsis.

Defective Ag-specific GC B cell differentiation and memory B cell and PC formation in CLP survivors. The impaired production of class-switched and high-affinity Ag-specific Abs seen in response to a TD immunization in CLP mice, despite the intact response to a TI $\mathrm{Ag}$, prompted us to assess the GC response after NP-CGG immunization. GCs are critical sites for SHM, where high-affinity B cells are selected and further differentiated into memory B cells and LLPCs. After immunization with NP-CGG, mice produce predominately $\mathrm{NP}$-specific Abs of the IgG1 isotype (27). We therefore enumerated GC and memory B IgG1 cells. The flow cytometry gating strategy used to identify NP-specific GC and memory B cells is depicted in Supplemental Figure 2. Flow cytometry revealed that the percentage of $\mathrm{NP}^{+} \mathrm{IgG1} 1^{+} \mathrm{GC} \mathrm{B}$ cells was significantly reduced in CLP mice compared with that in sham mice after immunization with NP-CGG (Figure 2A). To confirm an impaired GC B cell response in CLP mice, we performed sham or CLP surgery in S1pr2-ERT2cretdTomato (tdTomato ${ }^{+}$) reporter mice to irreversibly mark GC B cells with Tomato red (28) following treatment with tamoxifen. The flow cytometry gating strategy for tdTomato ${ }^{+} \mathrm{NP}$-specific IgG1 $1^{+}$ GC B cells and the quantification are shown in Supplemental Figure $3, \mathrm{~A}$ and $\mathrm{B}$. The percentage of $\mathrm{tdTomato}^{+} \mathrm{NP}^{+} \mathrm{IgG} 1^{+} \mathrm{GC}$ B cells was reduced in CLP mice compared with that in sham mice on day 14 after immunization (Supplemental Figure 3B), confirming a GC defect. We also analyzed $\mathrm{NP}^{+} \mathrm{IgG} 1^{+}$memory (non-GC) B cells. The percentage of these cells was significantly reduced in CLP mice
(Figure 2B) compared with that in sham mice. We next analyzed PCs. The gating scheme for NP-specific PCs is shown in Supplemental Figure 4. The percentage of $\mathrm{NP}^{+} \mathrm{IgG} 1^{+} \mathrm{CD} 138^{+} \mathrm{PCs}$ was strikingly diminished (Figure 2C) in immunized CLP mice compared with that in immunized sham mice after immunization.

The number of NP-specific I $g G$-secreting PCs is lower in immunized CLP mice. Having observed an impairment in NP-specific B cell differentiation into memory B cells and PCs in CLP mice by flow cytometry, we enumerated NP-specific Ab-secreting cells (ASCs) by ELISpot assay. As expected, and consistent with low serum antiNP-IgG titers, there were fewer NP-specific IgG ASCs, both lowaffinity $\left(\mathrm{NP}_{23}\right)$ and high-affinity $\left(\mathrm{NP}_{2}\right)$, in the spleens of CLP mice compared with sham mice at day 14 after immunization (Figure 3, A and B). We also examined LLPCs in the BM at day 56 after primary immunization. Both low- and high-affinity NP-specific IgG ASCs were almost absent in CLP mice and significantly less frequent than in sham mice (Figure 3, C and D).

Activation-induced cytidine deaminase and TNF- $\alpha m R N A$ expression in NP-specific GC B cells is reduced in CLP mice. Activationinduced cytidine deaminase (AID) enzyme activity is required for both class-switch recombination and SHM of Ig genes and generation of high-affinity B cell clones (29). We therefore measured the expression of AID in NP-specific GC B cells. We have shown that CLP mice exhibit reduced serum TNF- $\alpha$ compared with sham mice when challenged with LPS (30). Previous studies suggest that AID expression is inducible and can be regulated by TNF- $\alpha$ in an NF- $\kappa \mathrm{B}$-dependent manner (31). Therefore, we measured TNF- $\alpha$ mRNA expression in NP-specific GC B cells isolated from sham and CLP mice. NP-specific GC B cells from CLP mice exhibited a significant reduction in both AID (Figure 4A) and TNF- $\alpha$ (Figure 4B) transcripts compared with sham mice (Figure 4, A and B). Reduced AID expression in Ag-activated B cells in immunized CLP mice may be due to reduced TNF- $\alpha$ expression.

In addition to TNF- $\alpha$ expression in splenic B cells, mRNA expression for lymphotoxin $\alpha(\mathrm{LT} \alpha)$ and LT $\beta$, which are required for FDC development, was also analyzed. CLP mice and sham mice had equivalent levels of LT $\alpha$ and LT $\beta$ in NP-specific GC B cells (Figure 4, C and D).

Clusters of FDCs are differentially organized in CLP mice compared with sham mice. FDC clusters are the nidus for the GC response (32). Mature FDCs are key players in events such as class-switch recombination, SHM, and affinity maturation, processes that are required for efficient generation and selection of high-affinity Abs through direct interaction with GC B cells $(32,33)$. LT $\alpha$ and LT $\beta$ are critical to the generation of FDCs and TNF- $\alpha$ to the clustering of FDCs (34-37). Moreover, it has been shown that FDCs can induce AID expression in Ag-activated B cells (38). As NP-specific GC B cells exhibit reduced AID and TNF- $\alpha$ expression, we assessed the number of FDC clusters in the spleens of sham and CLP mice. Immunofluorescence staining with CD35, an FDC marker, revealed that the number of FDC clusters was reduced in CLP mice compared with that in sham mice (Figure 5, A and B). The gating strategy for FDC is shown in Supplemental Figure 5A. Flow cytometry showed no decrease in the percentage of FDCs (CD45-TER119- CD31-B220ICAM- $\left.1^{+} \mathrm{CD} 21 / \mathrm{CD} 35^{+}\right)$in the stromal cell population in CLP mice compared with that in sham mice (Supplemental Figure 5B), suggesting a dispersion of FDCs out of clusters. 
A

NP-CGG+alum

or
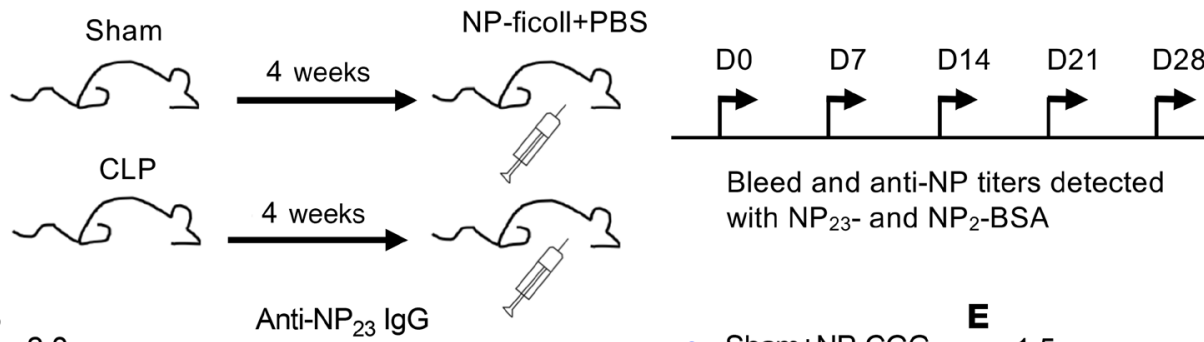

Bleed and anti-NP titers detected

with $\mathrm{NP}_{23}$ - and $\mathrm{NP}_{2}-\mathrm{BSA}$

B

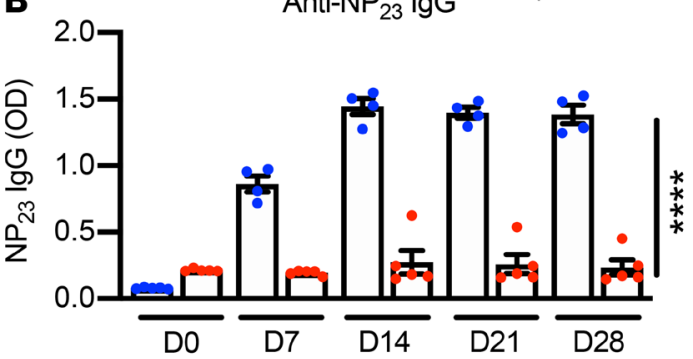

C

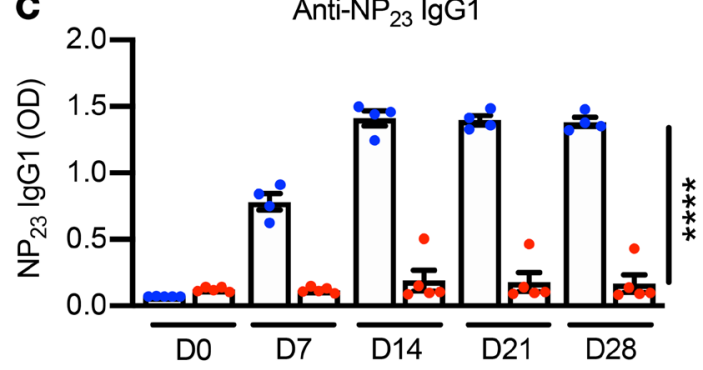

D

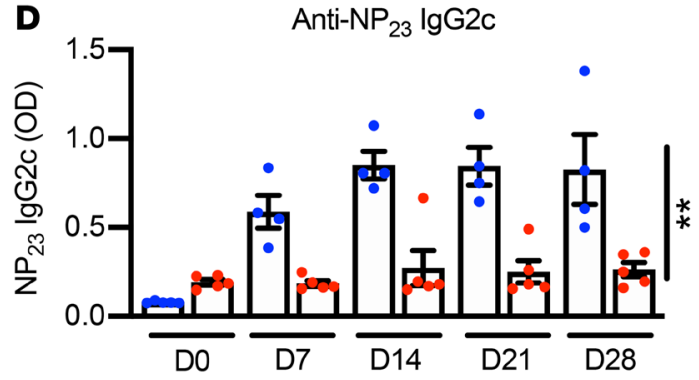

H

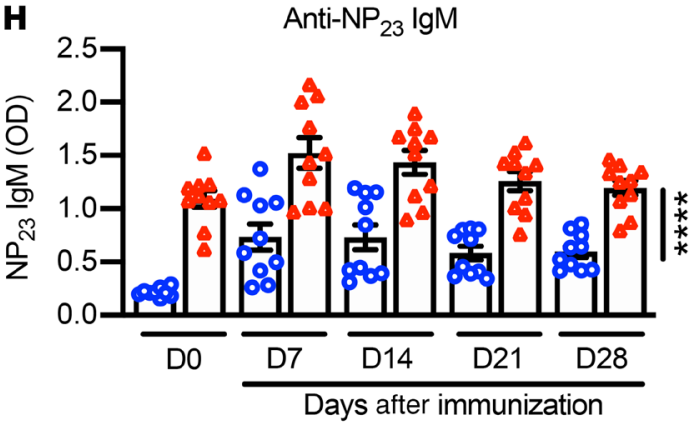

- Sham+NP-CGG

- $C L P+N P-C G G$

- Sham+NP-CGG

- $C L P+N P-C G G$
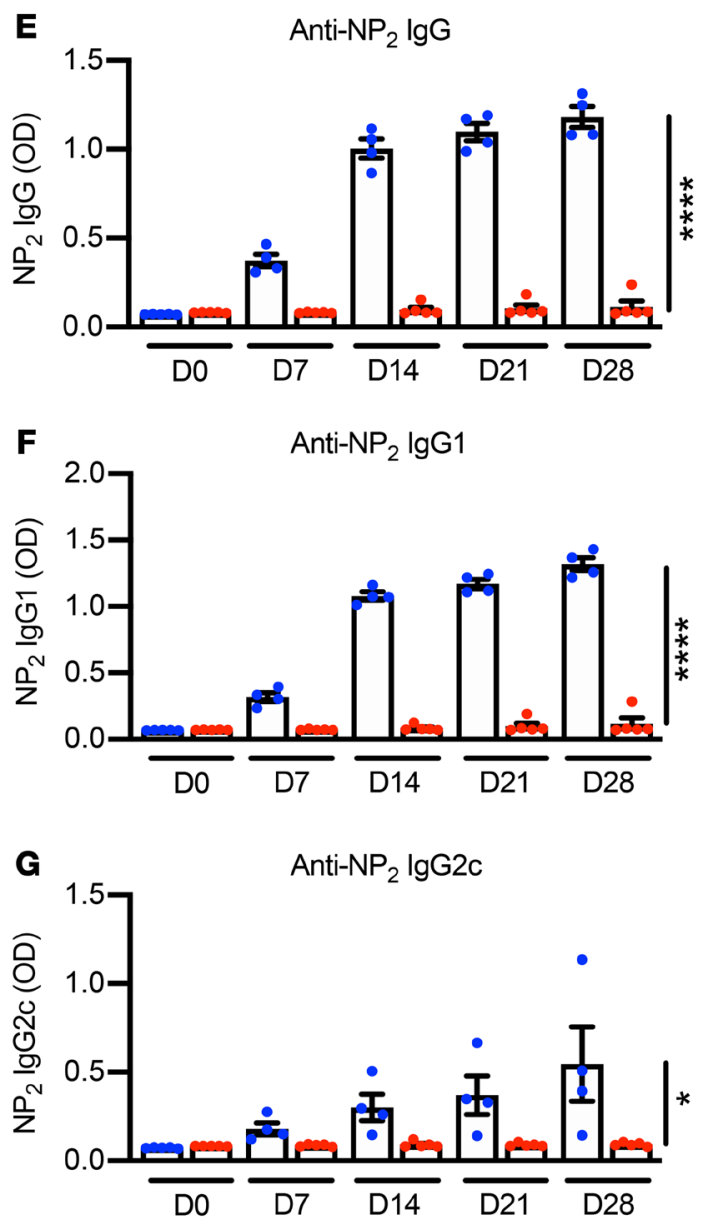

I

- Sham+NP-ficoll

$\Delta C L P+N P-$ ficoll

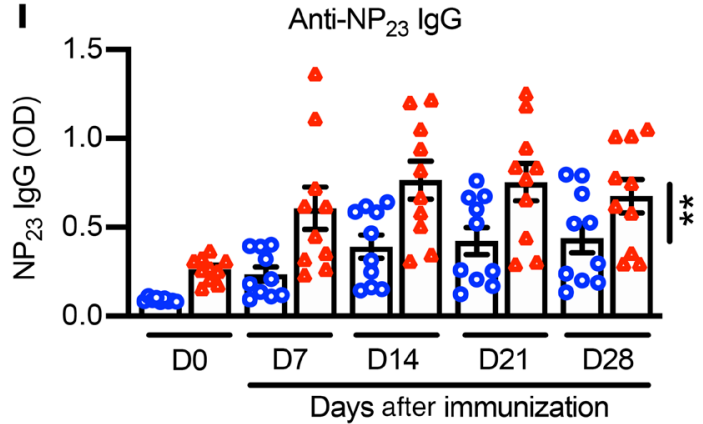

Figure 1. CLP survivors exhibit low levels of NP-specific TD IgG Ab after NP-CGG immunization and an intact response to TI Ag, NP-ficoll. Sham and CLP mice were immunized with NP-CGG in alum or NP-ficoll in PBS (single i.p. injection 4 weeks after surgery). Serum was analyzed for both low-affinity (NP ${ }_{23}$ ) and high-affinity $\left(\mathrm{NP}_{2}\right)$ anti-NP Abs by $\mathrm{NP}_{23}$ - and $\mathrm{NP}_{2}$-BSA ELISAs, respectively. (A) Experimental approach. Time-dependent response of (B) low-affinity antiNP-IgG, (C) -IgG1, and (D) -IgG2c and (E) high-affinity anti-NP-IgG, (F) -IgG1, and (C) -IgG2c levels after NP-CGG immunization ( $n=4-5$, Sham+NP-CGG; $n=5$, CLP+NP-CGC). (H) Low-affinity anti-NP 23 -IgM and (I) -IgG response after NP-ficoll immunization ( $n=9$, Sham+NP-ficoll; $n=10, C L P+N P$-ficoll). Data represent mean \pm SEM from 1 or 2 independent experiments. Sham+NP-CGG vs. CLP+NP-CGG, ${ }^{*} P<0.05,{ }^{* *} P<0.01$, and ${ }^{* * * *} P<0.0001$ or Sham+NP-ficoll vs. CLP+NPficoll, ${ }^{* *} P<0.01$ and ${ }^{* * * *} P<0.0001$ (mixed-effects model restricted maximum likelihood). 


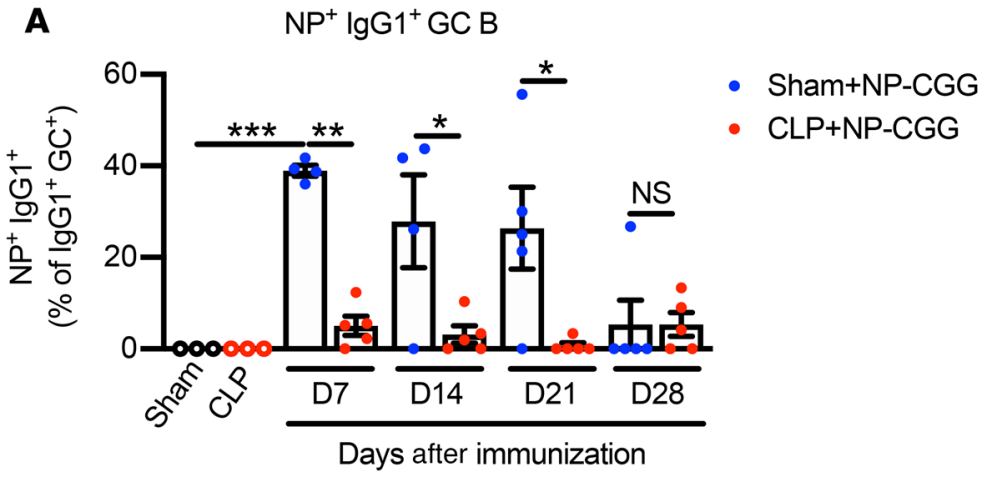

B $\quad \mathrm{NP}^{+} \operatorname{lgG} 1^{+}$non-GC B
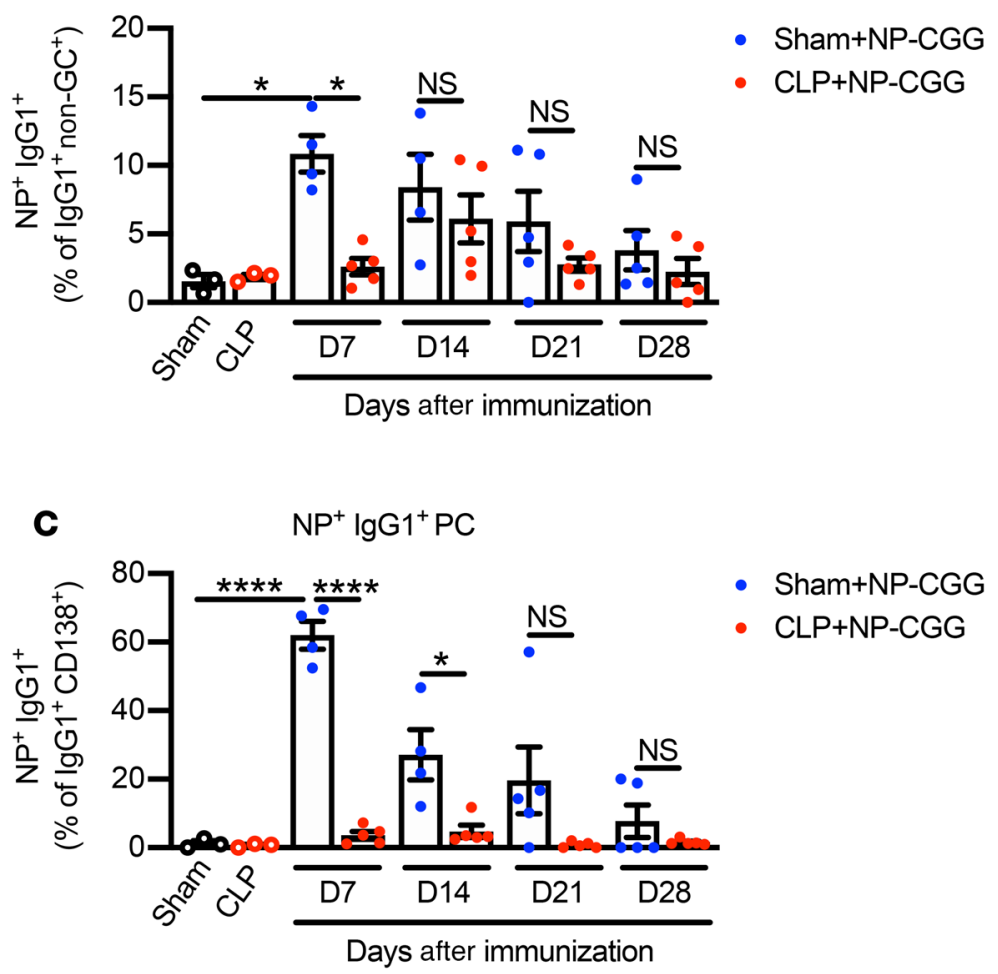

The binding of ICs on FDCs is reduced in CLP mice. Since the clustering of FDCs was impaired in CLP mice, we next examined whether their function was also impaired. One function of FDCs is to present native $\mathrm{Ag}$ in the form of ICs to high-affinity GC B cells for selection (15). To take advantage of intense phycoerythrin (PE) immunofluorescence, polyclonal anti-PE IgG was passively administered to mice, followed 1 day later by injection of $\mathrm{PE}$ to allow for the in vivo formation of ICs (Figure 5C). Immunofluorescence staining in spleen sections 24 hours after PE injection showed that less PE localized to FDCs $\left(\mathrm{CD} 35^{+}\right)$in CLP mice compared with sham mice, as assessed by colocalization of CD35 and PE (Figure 5, D and E), suggesting that FDCs may have lower expression of the CRs in CLP mice.

Altered gene expression in FDCs in CLP mice. To determine if the poor trapping of ICs on FDCs of CLP mice reflected reduced expression of CR2, we analyzed mRNA expression of CR2 in isolated FDCs. CR2 transcripts were significantly decreased in FDCs of
Figure 2. NP-specific GC and non-GC B cells and PCs are reduced in CLP mice. Sham and CLP mice were immunized with NP-CGC in alum (single i.p. injection 4 weeks after surgery). Quantification of the percentage of (A) NP+lgC1+ $\mathrm{GC}$ B cells, (B) NP+lgC1+ non-GC B cells, and (C) $\mathrm{NP}^{+} \mid \mathrm{gG} 1^{+} \mathrm{PCs}$ after 7, 14, 21, and 28 days after NP-CGG immunization ( $n=4-5$, Sham+NP-CGG; $n=5$, CLP+NP-CGG). Sham and CLP mice are unimmunized control mice injected with PBS $(n=3$, Sham; $n=3$, CLP). Data represent mean \pm SEM from 2 independent experiments. Sham vs. Sham+NP-CGG, ${ }^{*} P<0.05,{ }^{* * *} P<0.001$, and ${ }^{* * * *} P<0.0001$; Sham+NP-CGG vs. CLP+NP-CGG, ${ }^{*} P<0.05$, ${ }^{*} P<0.01$, and ${ }^{* * * *} P<0.0001$ (Tukey's post hoc 1-way ANOVA).

CLP mice compared with sham mice (Figure 6A). IC-FDC interactions lead to FDC activation and upregulation of ICAM-1, VCAM-1, and Fc $\gamma$ RIIB expression $(18,39)$. mRNA expression of ICAM-1 (Figure 6B), VCAM-1 (Figure 6C), and Fc $\gamma$ RIIB (Figure 6D) was also significantly reduced in FDCs of CLP mice compared with sham mice. TNFR1 (p55TNFR) on FDCs is necessary for development of a mature FDC network. IKK2 (IKK $\beta$ ) expression in FDCs is dispensable for the formation of FDC networks but indispensable for $\mathrm{B}$ cell affinity maturation and $\mathrm{Ab}$ production (39). Having observed reduced FDC clusters and impaired $\mathrm{Ab}$ affinity maturation in CLP mice, we analyzed mRNA expression of TNFR1 and IKK2. mRNA expression of TNFR1 and IKK2 in FDCs was significantly lower in CLP mice compared with that in sham mice (Figure 6, E and F). The maintenance of FDC phenotype and function requires constitutive signaling through LT $\beta R$ (40). mRNA expression of LT $\beta$ R in FDCs was significantly reduced in CLP mice compared with that in sham mice (Figure 6G). Taken together, these results suggest that there is a decreased expression of several genes critical to FDC function in CLP mice.

TNF- $\alpha$ neutralization results in impaired $A b$ response to TD Ag. To ask whether reduced TNF- $\alpha$ might be a major contributor to the impaired generation of a GC response in CLP mice, we treated WT mice with anti-TNF- $\alpha \mathrm{Ab}$ and immunized them with NP-CGG. We observed almost no FDC clusters in anti-TNF- $\alpha$-treated mice compared with isotype control-treated (Ctrl-treated) mice (Supplemental Figure 6A). NP-specific anti-NP-IgG (low- and high-affinity) levels were also significantly reduced in anti-TNF- $\alpha$-treated mice compared with isotype Ctrl-treated mice (Supplemental Figure 6, B and C).

$B$ cells from CLP mice are competent to respond to Ag. To ask whether sepsis-induced changes in B cells rendered them less capable of interacting with FDCs, we adoptively transferred B cells from sham or CLP mice to Rag1-KO mice along with CD4 ${ }^{+}$ $\mathrm{T}$ cells from WT C57BL/6J (Ctrl) mice. Twenty-one days after B and $\mathrm{T}$ cell transfer, we immunized Rag1-KO mice with NP-CGG. Rag1-KO mice given B cells from either sham or CLP mice exhibited an equivalent anti-NP-IgG response (Figure 7, A and B). Thus, $\mathrm{B}$ cells from CLP mice were not impaired in their ability to make anti-NP-IgG Ab (both low- and high affinity) compared with $\mathrm{B}$ cells from sham mice (Figure 7, A and B). In addition, Rag1-KO mice reconstituted with $\mathrm{B}$ cells from CLP mice showed restoration 
A

$\mathrm{NP}_{23}$ lgG splenocytes

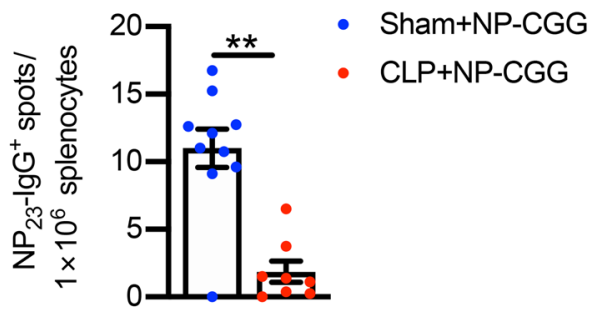

C $\mathrm{NP}_{23}$ lgG bone marrow

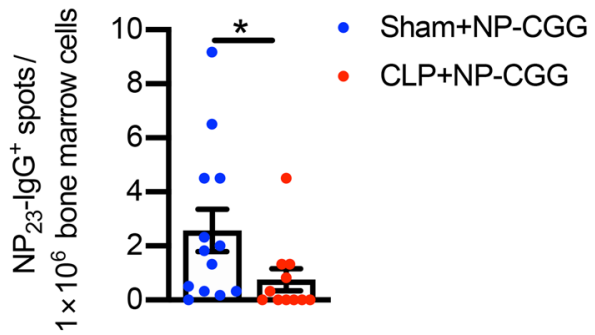

B

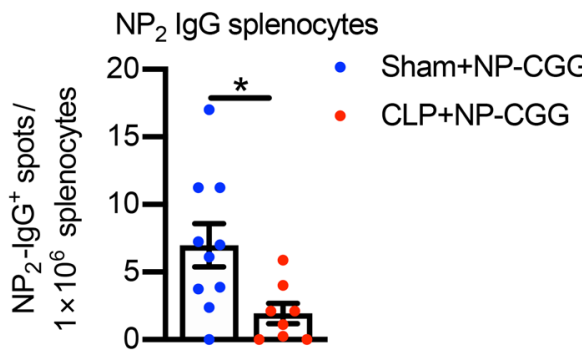

D $\quad \mathrm{NP}_{2} \lg \mathrm{G}$ bone marrow

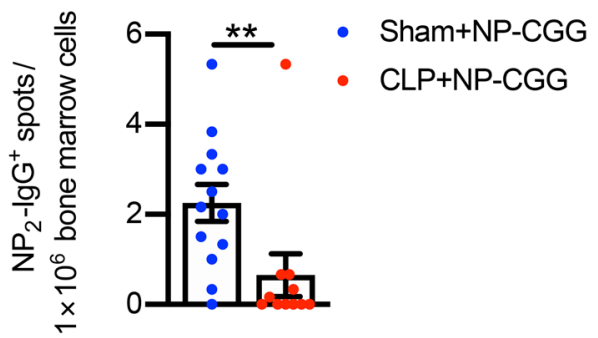

Figure 3. Numbers of NP-specific IgGsecreting $P C s$ are reduced in CLP mice. Sham and CLP mice 4 weeks after surgery were immunized with NP-CCG in alum (single i.p. injection) and cells were collected on days 14 (for splenocyte analysis) and 56 (for BM analysis) after primary immunization. ELISpot assays of anti-NP-IgG ASCs in the spleen and BM of immunized sham and CLP mice using $\mathrm{NP}_{23}$ - and $\mathrm{NP}_{2}$ BSA as coating Ags. (A) Low-affinity $\mathrm{NP}_{23}$-lgG+ spots and (B) high-affinity $\mathrm{NP}_{2}-$ $\mathrm{IgG} \mathrm{C}^{+}$spots in splenocytes at day 14 ( $n=10$, Sham+NP-CGG; $n=8$, CLP+NPCGC). (C) Low-affinity $\mathrm{NP}_{23}$-lgG+ spots and (D) high-affinity $\mathrm{NP}_{2}$-lgG+ spots in $\mathrm{BM}$ cells at day 56 after primary immunization $(n=$ 13, Sham+NP-CGG; $n=11$, CLP+NP-CGG). Data are shown as mean \pm SEM from 2 independent experiments. Sham+NP-CGG vs. CLP+NP-CGG, ${ }^{*} P<0.05$ and ${ }^{*} P<0.01$ (Mann-Whitney $U$ test). of FDC clusters similar to Rag1-KO mice reconstituted with B cells from sham mice (Figure 7, C and D). This result suggests that CLP mice have defective FDC function that fails to support the normal differentiation of $\mathrm{B}$ cells in a GC response.

\section{Discussion}

We have provided evidence in this study for the contribution of FDCs to the impaired Ag-specific GC B cell humoral responses in sepsis-surviving mice. We demonstrated that CLP mice are severely impaired in their ability to mount an Ab response on primary immunization with the TD Ag, NP-CGG, limiting their ability to produce high-affinity class-switched NP-specific Abs or to generate LLPCs and memory B cells. FDCs of CLP mice showed reduced retention of ICs. Of note, the transfer of B cells from CLP mice together with $\mathrm{T}$ cells from an unmanipulated mouse into Rag1-KO mice led to reconstitution of FDC clusters and an anti-NP-IgG response similar to that observed in sham mice following immunization. Thus, our data demonstrate that the impairment in TD humoral responses in CLP mice was not due to primarily intrinsic defects in the B cell compartment, but rather to FDC dysfunction. While a minority of CLP mice display persistent bacteria in the peritoneal cavity at the time of immunization, this cannot account for the impaired $\mathrm{Ab}$ response that was observed in all CLP mice.

Sepsis-associated mortality continues after the acute episode and hospital discharge (41). Adult sepsis-surviving patients have humoral immune impairments, as manifested by increased susceptibility to infections and a poor response to vaccinations for influenza or pneumococcus (42-45). Our data, therefore, may help explain the clinical scenario. CLP mice have a compromised humoral immunity when challenged with live influenza A virus (21). Therefore, reduced $\mathrm{Ab}$ response following $\mathrm{Ag}$ challenge in CLP mice is consistent with previously reported data $(20,21)$. We expanded on this observation showing that CLP mice can produce an enhanced response to TI immunization with NP-ficoll, corroborating that the defect is specific to a TD response.
Our results show that CLP mice have reduced GC response and Ig heavy chain class switching after TD immunization, resulting in minimal high-affinity IgG Ab production. Memory B cells and LLPCs persist for long periods of time in the BM generating life-long protective humoral immunity following infection and TD immune responses $(13,46)$. Our data show that NP-specific memory and PCs fail to increase in sepsis-surviving mice. A previous study (21) reported that reduced $\mathrm{Ab}$ production in CLP mice challenged with $\mathrm{Ag}$ is due, in part, to insufficient help from Tfh cells, leading to scarce B cell differentiation and class switching. It has now become apparent that presentation of membrane-bound Ag by FDCs plays a crucial role in $B$ cell activation, particularly during $B$ cell affinity maturation (8). The data we present here importantly extend our understanding of why there is an impaired response to TD immunization.

Our previous study (30) demonstrated that CLP mice exhibited reduced serum TNF- $\alpha$ following LPS challenge. B cells are the source of TNF- $\alpha$ that triggers FDC clustering and maturation (34). Our observation that TNF- $\alpha$ expression is reduced in CLP mice led us to investigate whether FDC clustering is impaired in CLP mice. Interestingly, we observed reduced numbers of FDC clusters in CLP mice compared with sham mice. In the GC, FDCs internalize Ag into a nondegradative cycling endosomal compartment where it is retained for extensive periods (47). as one of the important functions of FDCs is to present unprocessed Ag to B cells for selection, we tested the ability of FDCs to trap ICs in sham and CLP mice and found that FDCs were less effective at retaining ICs in CLP mice compared with sham mice. Our results indicate that the expression of CR2 and Fc $\gamma$ RIIB, both of which are responsible for trapping ICs, is lower in FDCs of CLP mice than sham mice, suggesting one possible reason why FDCs in CLP mice exhibit reduced trapping of ICs. However, the possibility cannot be omitted that FDCs in CLP mice may be preoccupied with microbial Ags, and increased competition among ICs may reduce presentation of a particular Ag.

TNFR1 is required for formation of the FDC network (39) and LT $\beta R$ for maintenance of mature FDCs $(34,48)$. It has been proposed 
A
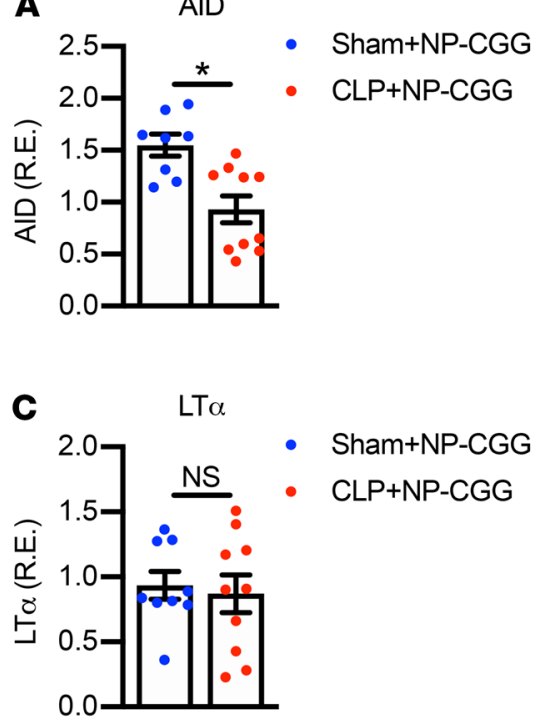

B

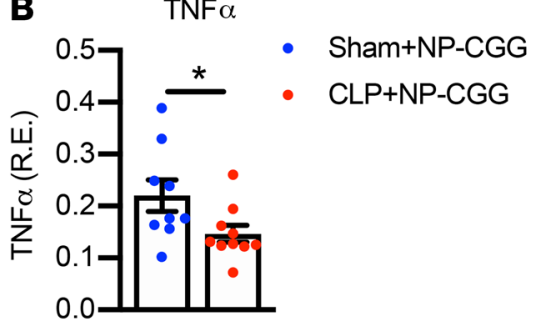

D

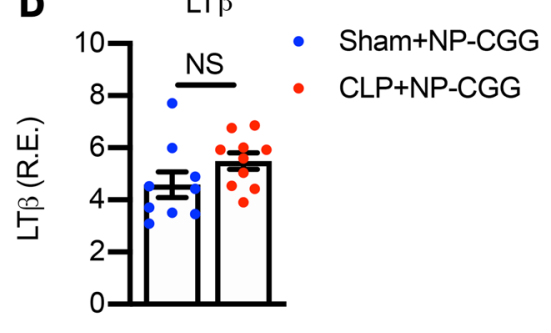

Figure 4. AID and TNF- $\alpha$ expression in NP-specific GC $B$ cells is reduced while there is no change in LT $\alpha$ and LT $\beta$ expression in immunized CLP mice.

(A) AID ( $n=8$, Sham+NP-CGG; $n=10$, CLP+NP-CGG), (B) TNF- $\alpha$, (C) LT $\alpha$, and (D) LT $\beta$ mRNA expression was assessed by qRT-PCR in flow-sorted NP+ GC B cells of sham and CLP mice at day 14 after immunization with NP-CGG ( $n=9$, Sham+NP-CGG; $n=10$, CLP+NP-CGG). Data represent mean \pm SEM from 3 independent experiments. Sham+NP-CGG vs. CLP+NP-CGG, ${ }^{*} P<0.05$ (Mann-Whitney $U$ test).

induction of Abs in CLP mice, we transferred $\mathrm{B}$ cells from CLP mice into Rag1-KO mice. Transfer of B cells from CLP mice led to a restoration of FDC networks and an intact primary B cell response following immunization. Since transfer of $B$ cells from CLP mice led to a restoration of FDC networks and an intact primary B cell response following immunization,

that signaling through TNF/TNFR1/TLR4 activates the classical or canonical NF-KB pathway involving the IKK2/NEMO complex, leading to nuclear translocation of p50:RelA heterodimers, while $\mathrm{LT} \beta \mathrm{R} / \mathrm{B}$ cell activating factor-R/CD40 signals through both classical and alternative or noncanonical IKK1/p100/NF-KB pathways (49). FDCs lacking IKK2 activated downstream of TNFR1 fail to upregulate adhesion molecules, such as ICAM- 1 and VCAM-1, impairing GC function (39). Our present findings suggest that reduced TNF/TNFR1 signaling leads to disrupted clusters of FDCs in CLP mice. We propose that the reduced expression of ICAM- 1 and VCAM- 1 that we observe on FDCs in CLP mice may be due to reduced expression of IKK2. ICAM- 1 and VCAM- 1 on FDCs are crucial for the physical association between FDCs and GC B cells (50), which enables FDCs to deliver survival signals to GC B cells (51). There is a possibility that reduced expression of these molecules in CLP mice may result in decreased survival of GC B cells. Previous studies have also shown that FDCs affect class switching and affinity maturation by promoting AID expression in GC B cells (38), and its expression is strongly reduced in FDCs lacking IKK2 (39). Of note, we showed that expression of AID is also reduced in GC B cells of CLP mice. As LT $\alpha$ signals through TNFR1, LT $\alpha$-mediated TNFR1 signaling may also be compromised in sepsis-surviving mice and may cooperate in mediating poor FDC clustering (52). While, we have not observed a change in LT $\alpha$ expression between sham and CLP mice, we suggest that TNFR1 reduction in FDCs is critical for impaired development of FDC networks in CLP mice. Buttressing this argument, we have further shown that $\mathrm{Ab}$ neutralization of TNF- $\alpha$ in normal mice results in loss of FDC clusters and thus impairs Ag-specific IgG responses comparable to what we observe in CLP mice. After the initial generation of FDCs, sustained signaling from LT $\beta R$ is required to maintain them in a fully differentiated and functional state (48). As we observed reduced expression of LT $\beta$ R in CLP mice, there is a possibility that CLP mice might not have FDCs with a fully matured phenotype.

It has been shown that FDCs fail to develop in Rag1-KO mice, where $\mathrm{B}$ and $\mathrm{T}$ cells are absent; however, the FDC network does develop following reconstitution of the mice with $\mathrm{B}$ and $\mathrm{T}$ cells (36, 53). To determine whether $B$ cells are responsible for the reduced the effect of sepsis on the subsequent $\mathrm{Ag}$-specific $\mathrm{Ab}$ responses is likely secondary to alterations in the microenvironment. Previously, we have shown that constitutive activation of vagus nerve signaling leads to reduced serum TNF- $\alpha$ responses following LPS challenge in CLP mice (30). This reflects a decrease in macrophage-derived TNF- $\alpha$. We speculate that sustained vagus nerve activation in CLP mice might be responsible for the low B cell TNF- $\alpha$ and impaired FDC function, leading to suppressed Ag-specific Ab responses.

In conclusion, we have shown here that FDC dysfunction leads to an immunosuppressed phenotype in sepsis-surviving mice.

\section{Methods}

Mice and immunization. C57BL/6J and Rag1-KO (B6.129S7-Rag1 < tm1Mom>/J) male mice (6-8 weeks old) were purchased from The Jackson Laboratory. S1pr2-ERT2cre (28) mice were provided by Tomohiro Kurosaki (Osaka University, Osaka, Japan) and bred with Rosa2 $6^{\text {Afl }}$ tdtomato mice (The Jackson Laboratory) at the Feinstein Institutes for Medical Research for generation of "S1pr2-ERT2cre-tdTomato mice." All mice were housed under specific pathogen-free conditions at room temperature $\left(22^{\circ} \mathrm{C}\right)$ and a 12-hour-light/dark cycle with access to chow diet and water. For immunizations, mice were immunized with $100 \mu \mathrm{g}$ $\mathrm{NP}_{20}$-CGG or CGG (Biosearch Technologies) precipitated in $100 \mu \mathrm{L}$ Imject alum (Thermo Scientific) or $50 \mu \mathrm{g} \mathrm{NP}_{55}$-ficoll (Biosearch Technologies) in $100 \mu \mathrm{L}$ PBS (Corning) by i.p. injection. Mice were sacrificed at the time points indicated in Results, and serum and cells were obtained. Serum was obtained by submandibular bleeding.

CLP procedure. Mice were subjected to CLP as described previously (30), with 30\%-50\% mortality. CLP was performed under isoflurane anesthesia, and the local anesthetic bupivacaine $0.25 \%(1-2 \mathrm{mg} /$ $\mathrm{kg}$ ) was administered by s.c. injection. A single dose of buprenorphine $(0.05 \mathrm{mg} / \mathrm{kg}$ s.c.) was administered to mice before midline incision. The cecum was isolated and ligated with 4-0 silk sutures below the ileocecal valve and then punctured once with a 22-gauge needle. Approximately $1 \mathrm{~mm}$ of feces was extruded, the cecum was placed back to the abdominal cavity, and then the abdominal muscle layer was closed with 6-0 PDS II sutures followed by skin layer closure with medical-surgical clips. In sham mice, the cecum was exposed but no ligation or puncture 
A

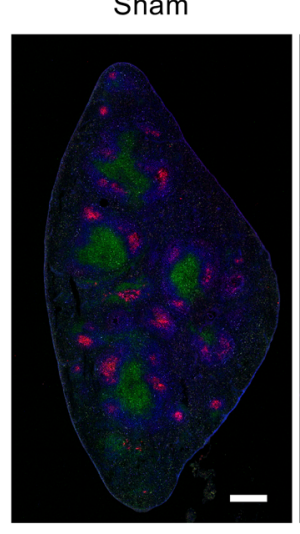

CD3 B220 CD35
B

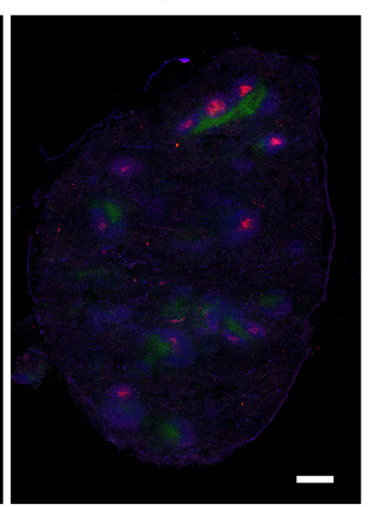

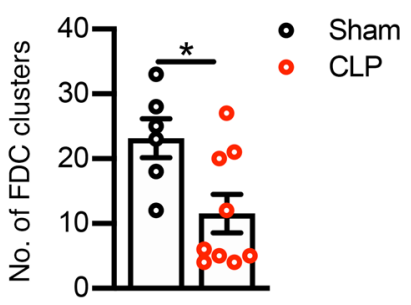

C

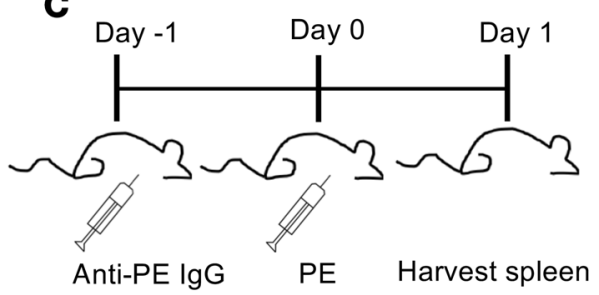

D
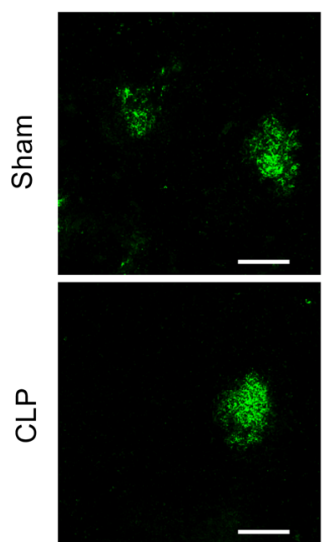

B220
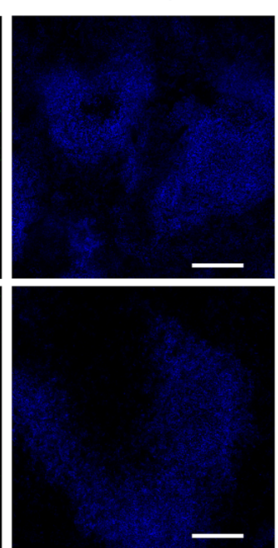

PE-IC

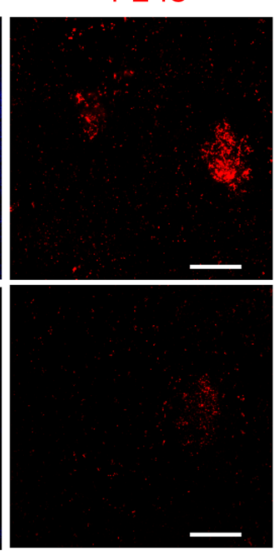

Merge

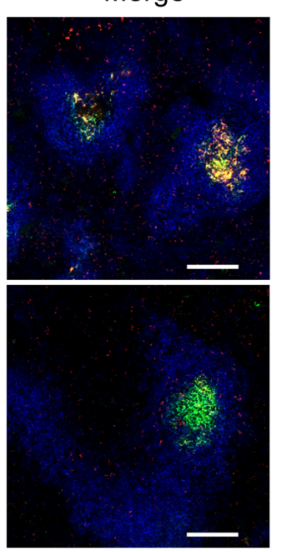

E

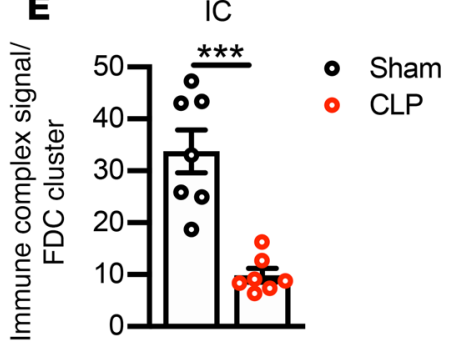

Figure 5. CLP mice have disrupted FDC clusters and reduced retention of ICs on FDCs. (A) Representative whole spleen sections from sham and CLP mice stained for CD3, B220, and CD35 6 weeks after surgery. Whole spleen images acquired by stitching tiled images. Original magnification, $\times 20$; scale bar: 500 um. (B) Quantification of FDC clusters from sham and CLP mice ( $n=6$, Sham; $n=9$, CLP). (C) Experimental approach. (D) Representative spleen sections showing localization of PE-IC (red) on FDC (green) after 24 hours of immunization. Original magnification, $\times 20$; scale bar: $200 \mu \mathrm{m}$. (E) Quantification of colocalization signal (yellow) in sham and CLP mice ( $n=7$, Sham; $n=7$, CLP). Data represent mean \pm SEM from 2 independent experiments. ${ }^{*} P<0.05$; ${ }^{* * *} P<0.001$ (Mann-Whitney $U$ test).

was performed. Both sham and CLP mice received 1 dose of antibiotics (imipenem/cilastatin; $0.5 \mathrm{mg} / \mathrm{kg}$ s.c.) and a single dose of sterile saline (0.5 mL/mouse s.c.) after surgery. The use of analgesics, antibiotics, and fluid resuscitation adhered to the MQTiPSS guidelines. Mice were monitored daily for survival and evaluated using the mouse grimace scale twice per day for the first 3 days followed by once per day for up to 7 days after surgery. At various time intervals, bacterial counts from blood (10-fold dilution in saline) and peritoneal lavage fluid $(2 \mathrm{~mL}$ of PBS) were determined by culturing $100 \mu \mathrm{L}$ aliquots of serial dilutions on agar plates containing $3.7 \% \mathrm{w} / \mathrm{v}$ brain-heart infusion broth (BD) and $1.5 \% \mathrm{w} / \mathrm{v}$ agar (BD). Plates were incubated at $37^{\circ} \mathrm{C}$ for 24 hours. Colonies were counted and are expressed as colony-forming units per $\mathrm{mL}$ blood or peritoneal lavage fluid (54).

ELISA and ELISpot assay. Serum levels of NP-specific or total Abs were measured by ELISA. Briefly, half-area 96-well flat-bottom plates (Corning) were coated with $10 \mu \mathrm{g} / \mathrm{mL} \mathrm{NP}_{2}$ - or $\mathrm{NP}_{23}$-BSA (Biosearch Technologies) or $5 \mu \mathrm{g} / \mathrm{mL}$ of goat anti-mouse IgG (Southern Biotech) in PBS overnight at $4^{\circ} \mathrm{C}$. Plates were then washed with $0.05 \%$ Tween 20 (Fisher Scientific) in PBS and blocked with 1\% BSA (Roche) in PBS for 1-2 hours at room temperature. Sera were diluted in $0.2 \%$ BSA in PBS (1:1500 for anti-NP-IgG, -IgG1, or -IgM; 1:100 for anti-NP-IgG2c;
1:20,000 for total $\operatorname{IgG}$ ) and incubated for 2 hours at room temperature. After washing, the plates were incubated with alkaline phosphatase-labeled (AP-labeled) goat anti-mouse IgG, IgG1, IgG2c, or IgM (1:1000; Southern Biotech) for 1 hour at room temperature, followed by detection using $1 \mathrm{mg} / \mathrm{mL}$ phosphatase substrate (MilliporeSigma) in substrate buffer (50 $\mathrm{mM} \mathrm{NaHCO}_{3}$, Fisher Scientific; $1 \mathrm{mM} \mathrm{MgCl}$, MilliporeSigma). The plates were read at $405 \mathrm{~nm}$ on a 1430 Multilabel Counter Spectrometer (PerkinElmer).

For ELISpot assays, 96-well Immulon 4HBX plates (Thermo Scientific) were coated with $10 \mu \mathrm{g} / \mathrm{mL} \mathrm{NP}_{2}$ - or $\mathrm{NP}_{23}$-BSA in PBS overnight at $4^{\circ} \mathrm{C}$. Plates were then washed and blocked with culture medium for 1 hour at $37^{\circ} \mathrm{C}$. Cells were added to wells as serial 1:2 dilutions in fresh culture medium consisting of RPMI (Gibco) with 10\% FBS, $100 \mathrm{U} /$ $\mathrm{mL}$ penicillin (Pen) and $100 \mu \mathrm{g} / \mathrm{mL}$ streptomycin (Strep) (both from HyClone), $10 \mathrm{mM}$ HEPES, and $55 \mu \mathrm{M} \beta$-mercaptoethanol (both from Gibco). Plates were briefly spun to settle cells to the bottom and incubated at $37^{\circ} \mathrm{C}$ in $5 \% \mathrm{CO}_{2}$ incubator overnight. After washing, plates were incubated biotinylated goat anti-mouse $\operatorname{IgG}$ (1:600; Southern Biotech) for 2 hours at $37^{\circ} \mathrm{C}$, followed by streptavidin-conjugated AP (1:1000; Southern Biotech) for 1 hour at $37^{\circ} \mathrm{C}$. For detection, 1 $\mathrm{mg} / \mathrm{mL}$ substrate (5-bromo-4-chloro-3-indolyl-phosphate, 4 -toluidine 
A
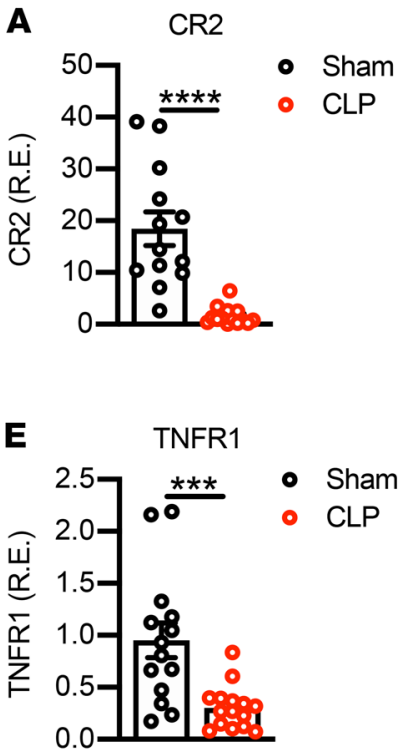
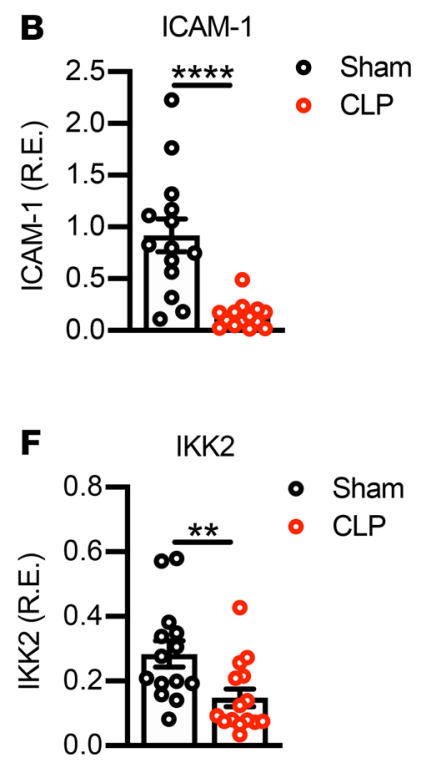

C
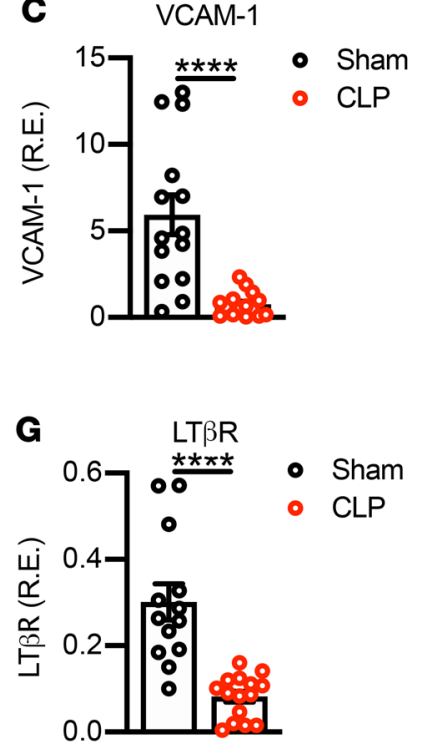

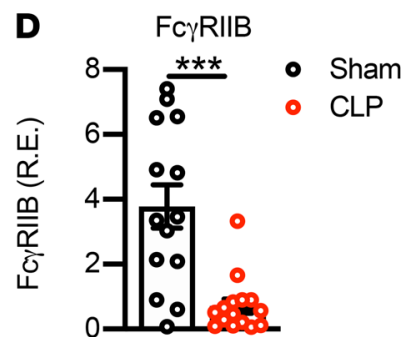

Figure 6. FDC gene expression is altered in CLP mice. (A) CR2 ( $n=13$, Sham; $n=14$, CLP), (B) ICAM-1 ( $n=14$, Sham; $n=14$, CLP), (C) VCAM-1 ( $n=14$, Sham; $n=15$, CLP), (D) Fc $\gamma$ RIIB ( $n=14$, Sham; $n=15$, CLP), (E) TNFR1 ( $n=14$, Sham; $n=15$, CLP), (F) IKK2 ( $n=14$, Sham; $n=15$, CLP), and (G) LT $\beta R$ ( $n=13$, Sham; $n=$ 15 , CLP) mRNA expression was assessed by qRT-PCR in flow-sorted FDCs of sham and CLP mice at 4 weeks after surgery. Data represent mean \pm SEM from 3 independent experiments. Sham vs. CLP, ${ }^{* *} P<0.01,{ }^{* * *} P<0.001,{ }^{* * *} P<0.0001$ (Mann-Whitney $U$ test).

salt, MilliporeSigma) in substrate buffer $(0.75 \mathrm{mM} \mathrm{MgCl} ; 0.01 \%$ Triton X-100, MilliporeSigma; 9.58\% 2-amino-methyl-1 propanol, MilliporeSigma) was used. After development of spots at room temperature, plates were rinsed with distilled $\mathrm{H}_{2} \mathrm{O}$ and air dried. Spots were counted manually under a light microscope (Olympus CK40-F100) (55).

Cell isolation. Single-cell suspensions were obtained from spleens as described previously (30) by standard procedures and red blood cells (RBC) were lysed using RBC lysis buffer (BioLegend). For FDC isolation, spleens were harvested from sham and CLP mice at 4 weeks after surgery, minced, and transferred to 6-well plates containing $2 \mathrm{~mL}$ of digestion medium (DMEM-high glucose [MilliporeSigma] + 2\% FBS + HEPES + Pen/Strep + Sodium pyruvate [Gibco] and $1 \mathrm{mg} / \mathrm{mL} \mathrm{Col-}$ lagenase D [MilliporeSigma]), $100 \mu \mathrm{g} / \mathrm{mL}$ DNase I (MilliporeSigma), and 0.6 U Dispase II (MilliporeSigma) and incubated for 15 minutes at $37^{\circ} \mathrm{C}$ in a humidified $\mathrm{CO}_{2}$ incubator. Tissue was pipetted up and down 10 times and incubated another 15 minutes with $2 \mathrm{~mL}$ of fresh digestion medium. Cell suspensions were passed through a $100 \mu \mathrm{m}$ nylon cell strainer (Fisher) and resuspended in complete DMEM containing 10\% FBS and $5 \mathrm{mM}$ ethylene-diaminetetraacetic acid (EDTA; Invitrogen). Cells were spun down and resuspended to $1 \times 10^{7}$ cells $/ \mathrm{mL}$ in magnetic activated cell sorting (MACS) buffer with $7 \mu \mathrm{L}$ anti-CD45 MACS microbeads (Miltenyi) and incubated for 15 minutes at $4^{\circ} \mathrm{C}$. Cells were washed with MACS buffer and resuspended again in MACS buffer and loaded onto a LS column (Miltenyi) to deplete CD $45^{+}$cells. After depletion of $\mathrm{CD} 45^{+}$cells, stromal cells were stained with Abs for phenotyping and sorting for further isolation of FDCs $(56,57)$.

$\mathrm{BM}$ cells were isolated as described previously (58) from both tibias and femurs of sham and CLP mice. Briefly, after removal of the condyles using a scissor to expose the metaphysis, the tibias and femurs were placed into $0.5 \mathrm{~mL}$ microcentrifuge tubes punched with 18-gauge needle at the bottom, and the tubes were nested in an intact $1.5 \mathrm{~mL}$ centrifuge tube, followed by centrifugation at 10,000 $\mathrm{g}$ for 15 seconds. The collected BM cells were used for ELISpot assay.
Flow cytometry and sorting. For GC or memory B cell and PC staining, splenocytes were first blocked with Fc block (rat anti-mouse CD16/CD32; clone 24. G2; BD Biosciences) for 5 minutes at room temperature. Cells were then incubated with anti-mouse Abs: BV421CD95 (clone Jo2; 1:100), FITC-GL7 (clone GL7; 1:100), PE-IgD (clone 11-26c.2a; 1:500), and PE-CD138 (clone 281-2; 1:500) (all from BD Biosciences); PE/Cy7-IgG1 (clone RMG1-1; 1:200; BioLegend); PerCP-Cy5.5-B220 (clone RA3-6B2; 1:300), PE-eFluor 610-IgM (clone II/41; 1:250), and Fixable Viability Dye-eFluor 506 (FVD-eFluor 506) (all from Thermo Fisher Scientific) in flow cytometry staining buffer (FACS) (HBSS containing 2\% FBS and $1 \mathrm{mM}$ EDTA) for 30 minutes on ice. Cells were washed twice with FACS buffer. FVD-eFluor 506 was added during staining with cell surface Abs. For intracellular staining, cells were fixed and permeabilized with the Transcription Factor Staining Buffer Set (eBioscience) according to the manufacturer's instructions. After permeabilization, cells were stained with anti-Ig Abs or $\mathrm{NP}_{6}$-BSA-biotin (Biosearch Technologies) dissolved in permeabilization buffer and incubated for 30 minutes on ice. Cells were washed and fixed in 1\% paraformaldehyde (MilliporeSigma). Flow cytometric acquisition was performed on a BD Fortessa (BD Biosciences). Analysis was performed using FACS Diva (BD Biosciences) and FlowJo software v10.6.1 (Tree Star).

Staining of NP-specific B cells from immunized mice was performed as previously described (59). Briefly, $\mathrm{NP}_{6}$-BSA-biotin was preincubated with streptavidin-APC (Thermo Fisher Scientific) on ice for 30 minutes, after which cells were incubated with $\mathrm{NP}_{6}$-BSA-biotin + streptavidin-APC on ice for 30 minutes.

For sorting of FDCs for mRNA expression studies, CD45- stromal cells were stained as described above with anti-mouse Abs: PerCP-CD45 (clone 30-F11; 1:100) and PE-Cy7-CD21/CD35 (clone 7E9; 1:200) (both from BioLegend); BV510-B220 (clone RA3-6B2; 1:100) and FITC-CD31 (clone MEC 13.3;1:200) (both from BD Biosciences); and PE-eFluor 610TER-119 (clone TER-119; 1:200), PE-ICAM-1 (clone YN1/1.7.4; 1:200), 
A

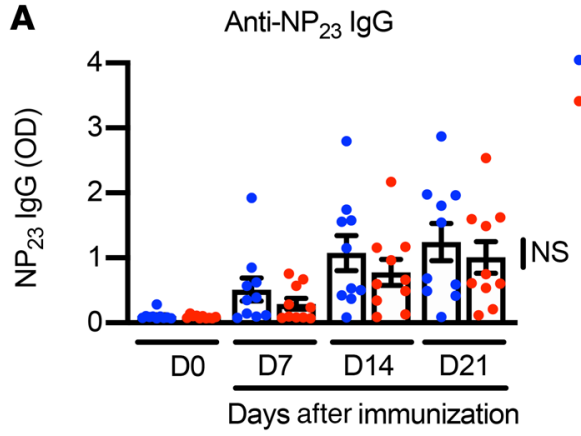

C

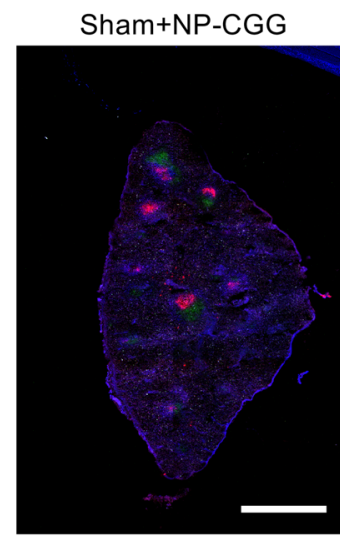

$C L P+N P-C G G$

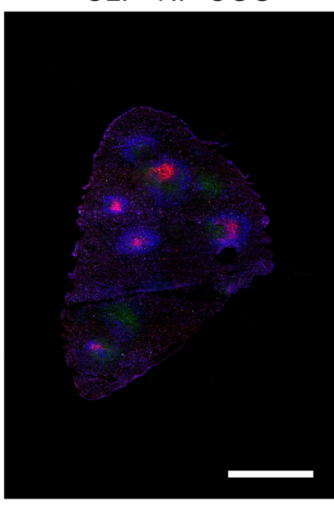

- Sham+NP-CGG

- CLP+NP-CGG
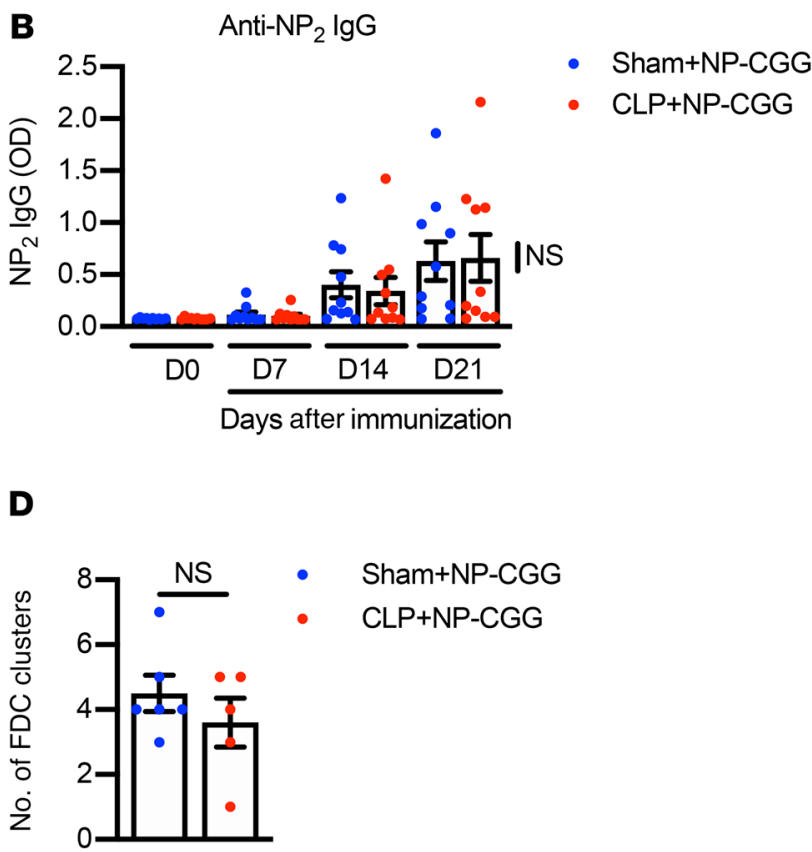

CD3 B220 CD35

Figure 7. B cell transfer from either sham or CLP mice into Rag1-KO mice leads to normal anti-NP Ab response and restoration of FDC clusters. B cells isolated from sham or CLP mice at 4 weeks after surgery together with CD4+ $T$ cells from WT mice were transferred into Rag1-KO mice. After 21 days of reconstitution, mice were immunized with NP-CGG in alum and serially bled up to day 21 of immunization. Time-dependent (A) low-affinity anti-NP ${ }_{23}$ lgG and (B) high-affinity anti-NP IgG response in Rag1-KO mice ( $n=10$, Sham+NP-CGG; $n=10$, CLP+NP-CGG). Data represent mean \pm SEM from 2 independent experiments. Sham+NP-CGG vs. CLP+NP-CGG (2-way repeated-measures ANOVA). Spleens were harvested at day 21 following immunization, and frozen sections were stained for CD3, B220, and CD35. (C) Representative whole spleen sections from Rag1-KO mice receiving B cells from sham or CLP mice. Whole spleen images acquired by stitching tiled images. Original magnification, $\times 20$; scale bar: $500 \mu \mathrm{m}$. (D) Quantification of FDC clusters in Rag1-KO mice ( $n=6$, Sham+NPCGG; $n=5$, CLP+NP-CGG). Data represent mean \pm SEM from 1 of 2 independent experiments. Sham+NP-CGG vs. CLP+NP-CGG (Mann-Whitney $U$ test).

and FVD-eFluor 780 (all from Thermo Fisher Scientific). FDCs were sorted with a $100 \mu \mathrm{M}$ nozzle into buffer containing HBSS with $20 \%$ FBS.

For sorting of NP-specific GC B cells for mRNA expression, total splenocytes were stained with Abs as described above. $\mathrm{NP}^{+} \mathrm{GC}$ B cells were isolated after sorting.

Cell sorting was performed on a FACS Aria (BD Biosciences). Freshly sorted NP-specific GC B cells or FDCs were pelleted and stored in TRIzol reagent (Life Technologies) at $-80^{\circ} \mathrm{C}$ until RNA isolation. The gating strategy for all panels and catalogs of Abs are provided in Supplemental Figures 2-5 and Supplemental Table 1.

Immunofluorescence. Spleens were fresh-frozen with dry ice, embedded in O.C.T. compound (Tissue-Tek; Fisher), and kept at $-80^{\circ} \mathrm{C}$ until processing. Cryosections were prepared at $10 \mu \mathrm{m}$ thickness using a Leica 3050s cryostat (Leica Biosystems Inc.) and air dried on glass slides. Slides were fixed in ice-cold acetone (Fisher) for 10 minutes and air-dried for 5 minutes. All incubations were performed in a humidified chamber. Slides were washed 3 times in PBS and blocked with 8\% normal goat serum (Abcam) for 30 minutes. Biotin-conjugated rat anti-mouse CD35 (clone 8C12; 1:100; BD Biosciences), APC-conjugated hamster antimouse CD3e (clone 145-2C11; 1:100; BD Biosciences), and eFluor 450conjugated rat anti-mouse B220 (clone RA3-6B2; 1:200; Thermo Fisher Scientific) were diluted in PBS containing 0.1\% Tween 20 and incubated overnight at $4^{\circ} \mathrm{C}$. The next day, slides were washed 3 times in PBS and incubated with streptavidin-conjugated Alexa Fluor 568 (strep-AF 568) (1:500; Thermo Fisher Scientific) for 45 minutes at room temperature. Slides were washed 3 times in PBS, dried, and mounted in Vectashield Antifade Mounting Medium (Vector Laboratories). Slides were visualized through a Zeiss LSM880 Confocal microscope. Images were analyzed and quantified by using the Zen Blue software (Zeiss).

In vivo generation of PE-ICs. Mice were passively immunized with $100 \mu \mathrm{g}$ rabbit anti-PE IgG (Rockland Immunochemicals) by retro-orbital i.v. injection. Twenty-four hours later, mice received $10 \mu \mathrm{g}$ B-PE (Invitrogen) i.p., and at 48 hours later (24 hours after injection of B-PE), mice were sacrificed, spleens were frozen and cut, and sections were stained as described above, with the exception that strep-AF 488 (1:500) was used instead of strep-AF 568 (60).

Treatments. tdTomato expression in S1pr2-ERT2cre-tdTomato mice was induced by i.p. administration of $2 \mathrm{mg}$ tamoxifen (MilliporeSigma) in $100 \mu \mathrm{L}$ of corn oil (MilliporeSigma) once per day for 3 consecutive days starting at day 11 after NP-CGG immunization (days 11-13). Mice were sacrificed at day 14 of immunization (28).

For in vivo neutralization of TNF- $\alpha$, LEAF-purified anti-mouse TNF- $\alpha$ (clone MP6-XT22) or rat IgG1, $\kappa$ isotype Ctrl (clone RTK2071) (BioLegend) $(250 \mu \mathrm{g}$ per mouse in $250 \mu \mathrm{L}$ PBS i.p.) was administered 
to WT C57BL/6J mice; 48 hours later, mice were immunized with NP-CGG $(100 \mu \mathrm{g})$ in alum $(100 \mu \mathrm{L})$, in a single i.p. dose. Mice received anti-mouse TNF- $\alpha$ or isotype Ctrl $(250 \mu \mathrm{g} / 250 \mu \mathrm{L}$ PBS, i.p.) 48 hour before and beginning on the day of immunization and on every third day until they were sacrificed at day 14 after immunization.

Adoptive transfer of $\mathrm{B}$ and $\mathrm{CD} 4^{+} \mathrm{T}$ cells. The purification of $\mathrm{B}$ and $\mathrm{CD}^{+} \mathrm{T}$ cells for reconstitution experiments was performed using Easysep mouse Pan-B cell isolation kit and EasySep mouse CD4 ${ }^{+} \mathrm{T}$ cell isolation kit, respectively (Stem Cell Technologies), according to the manufacturer's instructions. Purified B cells $\left(10 \times 10^{6}\right)$ from sham or CLP mice and $\mathrm{CD}^{+} \mathrm{T}$ cells $\left(2.5 \times 10^{6}\right)$ from WT C57BL/6J mice were transferred by retro-orbital i.v. injection into unirradiated Rag1KO mice. After 21 days of reconstitution, mice were immunized with NP-CGG precipitated in alum. Mice were serially bled after immunization to quantify anti-NP IgG response. Mice were sacrificed at day 21 of immunization, spleens were frozen and cut, and sections were stained as described above (36).

RNA isolation and cDNA synthesis. RNA isolation was performed using the Direct-zol RNA Microprep kit (Zymo Research) according to the manufacturer's instructions. Freshly isolated RNA was reverse transcribed into cDNA using the iScript cDNA Synthesis Kit (Bio-Rad) according to the manufacturer's instructions.

Quantitative real-time PCR. All TaqMan assays were purchased from Thermo Fisher Scientific. TaqMan assays used were as follows: TNF- $\alpha$ (Mm00443258_m1), AID (Mm01184115_m1), LT $\alpha$ (Mm00440228_gH), LT $\beta$ (Mm00434774_g1), CR2 (Mm00801681_ m1), Fc $\gamma$ RIIB (Mm00438875_m1), LT $\beta R$ (Mm00440235_m1), TNFR1 (Mm00441883_g1), IKK2 (Mm01222247_m1), VCAM-1 (Mm01320970_ m1), and ICAM-1 (Mm00516023_m1). cDNA was first subjected to preamplification for HPRT and respective genes using the TaqMan PreAmp Master Mix Kit (Thermo Fisher Scientific) according to the manufacturer's instructions. Real-Time PCR reactions were performed on a LightCycler 480 II (Roche) using master mix consisting of LightCycler 480 Probes Master (Roche), TaqMan assay, and cDNA PreAmp samples. Reactions were performed in duplicate. In all experiments, mRNA levels were normalized to the levels of the endogenous Ctrl HPRT (Mm03024075_m1), and relative expression was calculated by $2^{-\Delta \mathrm{Ct}}$.
Statistics. For comparisons of 2 groups, we used the Mann-Whitney $U$ test. For more than 2 groups, we used 1-way ANOVA with Tukey's post hoc test. For time course experiments (with multiple measurements for each mouse) without missing data, 2-way repeated-measures ANOVA was used. In cases where some data was missing, a mixed-effects model restricted maximum likelihood was performed. $P$ values of less than 0.05 were considered significant. Results are presented as the mean \pm SEM. Statistical analysis was performed with GraphPad Prism 8.

Study approval. All procedures were carried out in strict accordance with recommendations in the Guide for the Care and Use of Laboratory Animals (National Academies Press, 2011). The protocols were approved by the Institutional Animal Care and Use Committee of the Feinstein Institutes for Medical Research.

\section{Authorship contributions}

$\mathrm{MR}, \mathrm{BS}$, and BD designed the research, analyzed data, and wrote the manuscript. MR, ALB, and RL performed the experiments. MR and ALB performed CLP surgery. RL performed retro-orbital injections, submandibular bleeding, and breeding and maintenance of S1pr2-ERT2cretdTomato mice. BTV performed quantification of confocal images.

\section{Acknowledgments}

We thank Christopher Colon, Heriberto Borrero, and Barrett Wailing for flow cytometry, and Amanda Chan for confocal technical assistance. We thank Tomohiro Kurosaki for providing S1pr2-ERT2cre mice. The work was supported by a grant from the NIH (P01AI102852) to BS and BD and an American Association of Immunologists Careers in Immunology Fellowship to MR.

Address correspondence to: Betty Diamond, The Feinstein Institutes for Medical Research, Center for Autoimmune, Musculoskeletal and Hematopoietic Diseases, 350 Community Dr., Manhasset, New York, New York 11030, USA. Phone: 516.562.3830; Email: bdiamond@northwell.edu. Or to: Barbara Sherry, The Feinstein Institutes for Medical Research, Center for Immunology and Inflammation, 350 Community Dr., Manhasset, New York, New York 11030, USA. Phone: 516.562.3402; Email: bsherry@northwell.edu.
1. Singer M, et al. The third international consensus definitions for sepsis and septic shock (Sepsis-3). JAMA. 2016;315(8):801-810.

2. Torio CM, Andrews RM. Healthcare Cost and Utilization Project (HCUP) Statistical Briefs. Rockville (MD); 2006.

3. Quartin AA, et al. Magnitude and duration of the effect of sepsis on survival. Department of Veterans Affairs systemic sepsis cooperative studies group. JAMA.1997;277(13):1058-1063.

4. Wang HE, et al. Long-term mortality after community-acquired sepsis: a longitudinal population-based cohort study. BMJOpen. 2014;4(1):e004283.

5. Herran-Monge R, et al. Epidemiology and changes in mortality of sepsis after the implementation of surviving sepsis campaign guidelines. JIntensive Care Med. 2019;34(9):740-750.

6. Hotchkiss RS, et al. Sepsis-induced immunosuppression: from cellular dysfunctions to immunotherapy. Nat Rev Immunol. 2013;13(12):862-874.

7. Delano MJ, Ward PA. The immune system's role in sepsis progression, resolution, and long-term outcome. Immunol Rev. 2016;274(1):330-353.

8. Heesters BA, et al. Antigen presentation to B cells. Trends Immunol. 2016;37(12):844-854.

9. Noelle RJ, Snow EC. T helper cell-dependent B cell activation. FASEB J. 1991;5(13):2770-2776.

10. Allen CD, et al. Germinal-center organization and cellular dynamics. Immunity. 2007;27(2):190-202

11. Jacob J, et al. In situ studies of the primary immune response to (4-hydroxy-3-nitrophenyl)acetyl. I. The architecture and dynamics of responding cell populations. JExp Med.1991;173(5):1165-1175.

12. Allen CD, et al. Imaging of germinal center selection events during affinity maturation. Science. 2007;315(5811):528-531.

13. MacLennan IC. Germinal centers. Annu Rev Immunol. 1994;12:117-139.

14. Tarlinton D. B-cell memory: are subsets necessary? Nat Rev Immunol. 2006;6(10):785-790.

15. Kranich J, Krautler NJ. How follicular dendritic cells shape the B-cell antigenome. Front Immunol.
2016;7:225

16. Kosco-Vilbois MH. Are follicular dendritic cells really good for nothing? Nat Rev Immunol. 2003;3(9):764-769.

17. El Shikh ME, et al. Activation of B cells by antigens on follicular dendritic cells. Trends Immunol. 2010;31(6):205-211.

18. El Shikh ME, et al. Follicular dendritic cell (FDC)-FcgammaRIIB engagement via immune complexes induces the activated FDC phenotype associated with secondary follicle development. Eur J Immunol. 2006;36(10):2715-2724.

19. Park CS, Choi YS. How do follicular dendritic cells interact intimately with B cells in the germinal centre? Immunology. 2005;114(1):2-10.

20. Mohr A, et al. Sepsis leads to a reduced antigenspecific primary antibody response. Eur J Immunol. 2012;42(2):341-352.

21. Sjaastad FV, et al. Polymicrobial sepsis chronic immunoparalysis is defined by diminished Ag-specific T cell-dependent B cell responses. 
Front Immunol. 2018;9:2532.

22. Schmoeckel K, et al. Polymicrobial sepsis and non-specific immunization induce adaptive immunosuppression to a similar degree. PLoS One. 2018;13(2):e0192197.

23. Sjaastad FV, et al. Polymicrobial sepsis impairs antigen-specific memory CD4 T cell-mediated immunity. Front Immunol. 2020;11:1786.

24. Tinsley KW, et al. Sepsis induces apoptosis and profound depletion of splenic interdigitating and follicular dendritic cells. JImmunol. 2003;171(2):909-914.

25. Chatterjee S, et al. Oxidative stress induces protein and DNA radical formation in follicular dendritic cells of the germinal center and modulates its cell death patterns in late sepsis. Free Radic Biol Med. 2011;50(8):988-999.

26. Cuenca AG, et al. Cecal ligation and puncture. Curr Protoc Immunol. 2010; Chapter 19:Unit 193.

27. Takahashi $Y$, et al. In situ studies of the primary immune response to (4-hydroxy-3-nitrophenyl)acetyl. V. Affinity maturation develops in two stages of clonal selection. JExp Med.1998;187(6):885-895.

28. Shinnakasu R, et al. Regulated selection of germinal-center cells into the memory B cell compartment. Nat Immunol. 2016;17(7):861-869.

29. Luo Z, et al. The role of activation-induced cytidine deaminase in antibody diversification, immunodeficiency, and B-cell malignancies. J Allergy Clin Immunol. 2004;114(4):726-735

30. Rana $\mathrm{M}$, et al. Constitutive vagus nerve activation modulates immune suppression in sepsis survivors. Front Immunol. 2018;9:2032.

31. Duan Z, et al. AID expression increased by TNF- $\alpha$ is associated with class switch recombination of Ig $\alpha$ gene in cancers. Cell Mol Immunol. 2016;13(4):484-491.

32. Chaplin DD, Zindl CL. Taking control of follicular dendritic cells. Immunity. 2006;24(1):13-15.

33. Garin A, et al. Toll-like receptor 4 signaling by follicular dendritic cells is pivotal for germinal center onset and affinity maturation. Immunity. 2010;33(1):84-95.

34. Endres R, et al. Mature follicular dendritic cell networks depend on expression of lymphotoxin beta receptor by radioresistant stromal cells and of lymphotoxin beta and tumor necrosis factor by B cells. J Exp Med. 1999;189(1):159-168.

35. Allen CD, Cyster JG. Follicular dendritic cell networks of primary follicles and germinal centers: phenotype and function. Semin Immunol. 2008;20(1):14-25.

36. Fu YX, et al. B lymphocytes induce the formation of follicular dendritic cell clusters in a lymphotoxin alpha-dependent fashion. J Exp Med. 1998;187(7):1009-1018.

37. Fu YX, Chaplin DD. Development and maturation of secondary lymphoid tissues. Annu Rev Immunol. 1999;17:399-433.

38. Aydar Y, et al. The influence of immune complex-bearing follicular dendritic cells on the IgM response, Ig class switching, and production of high affinity IgG. JImmunol. 2005;174(9):5358-5366.

39. Victoratos P, et al. FDC-specific functions of p55TNFR and IKK2 in the development of FDC networks and of antibody responses. Immunity. 2006;24(1):65-77.

40. Boulianne B, et al. AID-expressing germinal center $\mathrm{B}$ cells cluster normally within lymph node follicles in the absence of FDC-M1+ CD35+ follicular dendritic cells but dissipate prematurely. J Immunol. 2013;191(9):4521-4530.

41. Delano MJ, Ward PA. Sepsis-induced immune dysfunction: can immune therapies reduce mortality? J Clin Invest. 2016;126(1):23-31.

42. Martin S, et al. Sepsis and immunosenescence in the elderly patient: a review. Front Med (Lausanne). 2017;4:20.

43. de Bourcy CF, et al. Phylogenetic analysis of the human antibody repertoire reveals quantitative signatures of immune senescence and aging. Proc Natl Acad Sci U S A. 2017;114(5):1105-1110.

44. Ekdahl K, et al. Immunoglobulin deficiencies and impaired immune response to polysaccharide antigens in adult patients with recurrent community-acquired pneumonia. Scand J Infect Dis. 1997;29(4):401-407.

45. Shankar-Hari M, et al. Endogenous IgG hypogammaglobulinaemia in critically ill adults with sepsis: systematic review and meta-analysis. Intensive Care Med. 2015;41(8):1393-1401.

46. McHeyzer-Williams LJ, McHeyzer-Williams MG. Antigen-specific memory B cell development. Annu Rev Immunol. 2005;23:487-513.

47. Heesters BA, et al. Endocytosis and recycling of immune complexes by follicular dendritic cells enhances $\mathrm{B}$ cell antigen binding and activation.
Immunity. 2013;38(6):1164-1175.

48. Krautler NJ, et al. Follicular dendritic cells emerge from ubiquitous perivascular precursors. Cell. 2012;150(1):194-206.

49. Bonizzi G, Karin M. The two NF-kappaB activation pathways and their role in innate and adaptive immunity. Trends Immunol. 2004;25(6):280-288.

50. Koopman G, et al. Adhesion through the LFA-1 (CD11a/CD18)-ICAM-1 (CD54) and the VLA-4 (CD49d)-VCAM-1 (CD106) pathways prevents apoptosis of germinal center B cells. JImmunol. 1994;152(8):3760-3767.

51. Park CS, et al. Follicular dendritic cells produce IL-15 that enhances germinal center B cell proliferation in membrane-bound form. JImmunol. 2004;173(11):6676-6683.

52. Drayton DL, et al. I kappa B kinase complex alpha kinase activity controls chemokine and high endothelial venule gene expression in lymph nodes and nasal-associated lymphoid tissue. JImmunol. 2004;173(10):6161-6168.

53. Kapasi ZF, et al. Induction of functional follicula dendritic cell development in severe combined immunodeficiency mice. Influence of $\mathrm{B}$ and $\mathrm{T}$ cells. JImmunol. 1993;150(7):2648-2658.

54. Delano MJ, et al. MyD88-dependent expansion of an immature GR-1(+)CD11b(+) population induces $\mathrm{T}$ cell suppression and $\mathrm{Th} 2$ polarization in sepsis. J Exp Med. 2007;204(6):1463-1474.

55 . Suurmond J, et al. Loss of an IgG plasma cell checkpoint in patients with lupus. J Allergy Clin Immunol. 2019;143(4):1586-1597.

56. Sato K, et al. Improved protocol for the isolation of naive follicular dendritic cells. Mol Immunol. 2016;78:140-145.

57. Rodda LB, et al. Single-cell RNA sequencing of lymph node stromal cells reveals niche-associated heterogeneity. Immunity. 2018;48(5):10141028.e6.

58. Amend SR, et al. Murine hind limb long bone dissection and bone marrow isolation. J Vis Exp. 2016;(110):53936.

59. Suurmond J, et al. Patterns of ANA+B cells for SLE patient stratification. JCI Insight. 2019;4(9):e127885.

60. Heesters BA, et al. Follicular dendritic cell isolation and loading of immune complexes. Methods Mol Biol. 2017;1623:105-112. 